\title{
Adults' Reports of the Role of Psychotherapy in Integrating Their Childhood Near-Death Experiences: A Preliminary Investigation
}

\author{
Jenny R. Moores, Ph.D., and Sue Ammen, Ph.D. \\ Alliant International University
}

\begin{abstract}
The purpose of this exploratory research was to learn if adults who had childhood near-death experiences (NDEs) sometime between pre-birth to age 17 years had psychotherapy and if they believed it helped them achieve psychological integration of their NDEs. Participants completed three instruments: the NDE Scale (Greyson, 1990), the author-developed Childhood NDE and Psychotherapy Questionnaire, and the three Subjective and Psychological Well-Being Scales (Diener \& Biswas-Diener, 2008). Of 29 respondents, 23 met the NDE Scale criteria for an NDE. Results for the $15(67 \%)$ who had engaged in psychotherapy showed statistically significant correlations with large effects between psychological integration of NDEs and more positive emotional feelings $(r=.77, p<.01)$ and fewer negative emotions $(r=-.84, p<.01)$. The correlation between psychological integration of NDE and success of psychotherapy in facilitating NDE integration was not statistically significant with a small effect $(r=.16, p>.10)$. The psychotherapy factors identified by participants as successful in helping them process and integrate their NDEs included having a therapist who accepted the NDE as real and validated the experience and who helped the NDEr express thoughts and feelings about, explore the meaning of, and resolve any guilt around the NDE. Results supported the idea that psychological
\end{abstract}

Jenny R. Moores, Ph.D., experienced near-death as an eight-year-old child during hospitalization for an intestinal virus. She completed the research described in this article as part of her doctoral dissertation at the California School of Professional Psychology, Alliant International University, Fresno, CA. She is now a researcher and Licensed Professional Counselor in Blanco, Texas. Dr. Moores wishes to thank the International Association for Near-Death Studies and the American Center for the Integration of Spiritually Transformative Experiences (ACISTE) for guidance that provided a wealth of knowledge. This article is based in part on Dr. Moores' presentation at the inaugural conference of ACISTE in October of 2012.

Sue Ammen, Ph.D., is a Professor at the California School of Professional Psychology, Alliant International University, Fresno, CA.

Correspondence regarding this article should be sent to Dr. Moores at e-mail: jennymooresphd@gmail.com. 
integration of NDEs is related to subjective and psychological wellbeing, and they provided clues about features of psychotherapy that might promote NDE integration. Limitations of the study, implications of results for psychotherapeutic interventions, and suggestions for future research are discussed.

KEY WORDS: near-death experience, childhood, psychotherapy, counseling, spirituality

The American Center for Spiritually Transformative Experiences (2010) asserted that

an experience is spiritually transformative when it causes people to perceive themselves and the world profoundly differently: by expanding the individual's identity, augmenting their sensitivities, and thereby altering their values, priorities and appreciation of the purpose of life. This may be triggered by surviving clinical death, or by otherwise sensing an enlarged reality ... [such as] near-death experiences (NDEs) [and] near-death-like experiences (NDLEs) . . . STEs include or may be called numinous, noetic, transcendent, transpersonal, mystical, anomalous, religious, paranormal, parapsychological or ecstatic experiences ... STEs can be catalysts for permanent and dramatic changes ... Many also involve difficult challenges for the experiencer before integration of the experience into one's life is complete. (para. 1, 2, \& 4)

An estimated 8 million Americans have experienced one specific type of STE, childhood NDEs (Gallup \& Proctor, 1982). Bruce Greyson (as cited in International Association for Near-Death Studies, 2009) defined an NDE as

a profound psychological event that may occur to a person close to death or, if not near death, in a situation of physical or emotional crisis. Because it includes transcendental and mystical elements, an NDE is a powerful event of consciousness; it is not mental illness. (p. 1)

Extensive research has now firmly established a pattern of NDE aftereffects that manifest psychologically, spiritually, physically, and socially and that often are challenging for experiencers as well as their intimates and associates (Flanagan, 2008; International Association of Near-Death Studies, 2010; Noyes, Fenwick, Holden, \& Christian, 2009). Researchers have found that these aftereffects and challenges characterize near-death experiencers (NDErs) of all ages but may be particularly challenging for childhood NDErs because of their less developed egos and limited life experience (Atwater, 1996; Sutherland, 
2009). Extremely little is known about the frequency and effectiveness of psychotherapy in the NDE integration process among NDErs in general and child NDErs in particular (Foster, James, \& Holden, 2009).

The purpose of this research was to learn if adult childhood NDErs reported having had psychotherapy and if they believed it helped them achieve psychological integration of their NDEs. For the purpose of this study, psychological integration is defined as a person being fully aware of one's experience without creating blocks in one's current functioning (Seeman, 2008).

Adult childhood NDErs were adult participants who reported having experienced an NDE any time during an age range from pre-birth to age 17 years. Hypotheses included a direct relationship between (a) self-reported psychological integration of childhood NDEs and selfreported subjective and psychological wellbeing outcomes, and (b) selfreported psychological integration and self-reported successful psychotherapy. In addition, content and types of psychotherapy were explored with the adult childhood NDErs to learn what they believed would be helpful with their NDE integration processes.

\section{Method}

This study was approved by the Institutional Review Board at Alliant International University.

\section{Participants}

Participants were recruited from an International Association for Near-Death Studies (IANDS) conference, IANDS e-mail lists, and word-of-mouth communication between NDErs. Criteria for initial inclusion in the study were age 18 years or older, fluency in English, and a self-reported NDE prior to age 18 years. All 29 participants who met these criteria completed an assessment battery that included the NDE Scale (Greyson, 1990), a psychometrically sound instrument used widely in NDE research to assess the presence (score 7 or higher) and depth (higher score indicating deeper) of an NDE; it is further described below. Six participants scored 6 or lower and comprised the near-death-like experience (NDLE) group. The 23-member NDE group was almost evenly split between males (48\%) and females (52\%), and the majority (82\%) was Caucasian (U.S.). NDErs' ages ranged from 33 to 75 years, with over half in their 50 s $(M=53.1, S D=10.8)$. Educa- 
tion of NDE group members was approximately equally distributed across levels ranging from high school education to doctoral degree.

The NDLE group consisted of $100 \%$ males and 67\% Caucasian (U.S.). Group members ranged in age from 33 to 72 years $(M=61.2$, $S D=14.5)$ and were predominantly in their 60 s or older $(85 \%)$. The NDE and NDLE groups were compared on selected demographic data; given the very small NDLE sample size, results are only tentative. With $52 \%$ females and $48 \%$ males in the NDE group, but $100 \%$ males in the NDLE group, the gender patterns were notably different. The majority in both groups was Caucasian (U.S.). Though the mean age of the NDLE group was older than that of the NDE group, the difference was not significant, $t(27)=1.53, p=.14)$.

\section{Instruments and Analysis}

Participants completed three instruments either online or in paper form: the NDE Scale (Greyson,1990), the first-author-developed Childhood NDE and Psychotherapy Questionnaire, and the Subjective and Psychological Well-Being Scales (Diener \& Biswas-Diener, 2008). The online form was created using Qualtrics, a secure, password-protected online survey program. The paper form was either given to and returned from the participant in person or sent to and returned from the participant via U.S. Postal Service.

The Childhood NDE and Psychotherapy Questionnaire (CNPQ) was a self-report instrument designed to assess past experiences with psychotherapy and explore possible future therapeutic interventions that could be utilized for the benefit of individuals who had an NDE as a child. It consisted of multiple questions and short answer style questions divided into two sections. Section I was subdivided into two subgroups: (a) demographics, NDE, and awareness of NDEs; and (b) disclosure of NDE, experience of psychotherapy, and psychological integration. Section II was subdivided into two subgroups: (a) beliefs and attitudes about psychotherapy for childhood NDErs, and (b) types of groups and therapy suggested for childhood NDErs. Participants who reportedly had not had psychotherapy skipped Section I and completed Section II. The CNPQ was not assessed for validity or reliability.

The NDE Scale was designed by Bruce Greyson (1983) to assess presence and depth of an NDE. The Scale was originally designed as a 33-item scaled-response questionnaire that Greyson (1990) subsequently refined into a shorter 16 -item version. It is comprised of four sets of four questions that identify cognitive, affective, paranormal, 
and transcendental NDE features. Total possible score ranges from zero to 32 ; for research purposes, a minimum score of 7 indicates an NDE. The Scale was found to have good internal consistency, splithalf reliability, and test-retest reliability (Greyson, 1983, 1990, 2007; Lange, Greyson, \& Houran, 2004).

The three Subjective and Psychological Well-Being Scales (SPWB) were developed by Diener and Biswas-Diener (2008) to be used in conjunction or separately for research and clinical purposes to assess emotional wellbeing. According to the SPWB Scales authors, subjective wellbeing refers to how people rate their lives based on terms of global judgment; domains of the person's life such as work or domestic life; and emotional states or feelings, either positive or negative. The three component scales are the Satisfaction With Life Scale (SWLS) to measure "global life satisfaction" (Diener, Emmons, Griffin, \& Larson, 1985, p.71); the Emotional Wellness Scale (EWS) to measure current emotions with three subscales-Pleasant Feelings, Unpleasant Feelings, and a Happiness Balance score that is the difference between pleasant and unpleasant feeling scores; and the Psychological Flourishing Scale (PFS) to measure psychological wealth-a person's overall attitude, goals, and engaging attitude toward life.

Hypotheses were tested on the NDE group using Pearson product moment correlations. The criterion for statistical significance was set at $p<.05$. In the absence of norms for effect size in NDE research, the strength of the relationship effect size index $(r)$ was defined as .1 for small, .3 for medium, and .5 for large, based on Jacob Cohen's (1992) power primer.

\section{Results}

\section{NDE Integration, Wellbeing, and Success of Psychotherapy}

Only the NDEr participants who indicated they had participated in psychotherapy were asked the NDE integration question; therefore, the following results are limited to those participants $(n=15)$. They were asked to rate the extent to which they felt they had integrated their childhood NDEs. Sixty-seven percent of these childhood NDErs reported that they felt they had psychologically integrated their NDEs and that it was an important part of who they are. The remaining NDErs reported feeling mostly integrated (13\%), somewhat integrated (7\%), and not at all or don't know (13\%). 
The first research question addressed the relationship between NDE integration and various aspects of subjective and psychological wellbeing as measured by the SPWB scales. Results showed statistically significant correlations with large effects between psychological integration of NDE and more positive emotional feelings $(r=.77$, $p<.01)$ and fewer negative emotions $(r=-.84, p<.01)$. The relationship with Satisfaction With Life Scale (SWLS) also yielded a large effect at $r=.50$, but did not reach significance $(p<.10)$. The relationship between NDE Integration and Psychological Flourishing (PFS) yielded a medium effect but also was not significant $(r=.44, p>.10)$. The results from these correlations support the idea that psychological integration of NDE is related to positive emotional wellness.

The second research question addressed the relationship between reported NDE integration and success of psychotherapy. When the 15 NDErs who had attended psychotherapy were asked, "Overall, do you believe psychotherapy was successful in helping you process and integrate your NDE?" their responses ranged from 1 (Not Helpful or Don't Know) to 5 (Yes, Very Helpful) with a mean score of $1.6(S D=1.5)$. The relationship between reported NDE Integration and success of psychotherapy to facilitate that integration was not significant with a small effect $(r=.16, p>.10)$. Only $27 \%(n=4)$ of the subjects who had participated in therapy reported that psychotherapy was mostly or very helpful in supporting their psychotherapy integration. However, all of those who reported the psychotherapy as helpful also reported that they had psychologically integrated the NDE into their current life functioning. We next examined what contributes to successful psychotherapy for individuals who have experienced an NDE.

\section{Factors in Successful Psychotherapy that Support NDE Integration}

The 15 NDEr psychotherapy participants indicated the degree and manner in which their NDEs were addressed in their psychotherapy, from not at all (1), somewhat (2), quite a bit (3), to yes (4). These factors included whether the therapist was aware of the NDE(s), whether the therapist accepted the NDE as real, and whether the NDE was a focus of treatment. They were also asked whether the mental health professional had helped them (a) express their feelings and thoughts about the NDE and how it had affected them, (b) acknowledge and validate the experience as real for them, (c) resolve any guilt that may have 
arisen for them related to the NDE, and (4) explore the meaning of the NDE in understanding themselves and their experiences (see Table 1).

Table 1

Therapist Factors in Addressing NDEs and Correlations with Treatment Success

\begin{tabular}{llc}
\hline \multicolumn{1}{c}{ Therapist Factors ${ }^{1}$} & $M(S D)$ & $\begin{array}{c}\text { Correlation } \\
\text { with Treatment } \\
\text { Success }^{2}\end{array}$ \\
\hline $\begin{array}{l}\text { Therapist helped: } \\
\text { Explore meaning of NDE }\end{array}$ & $2.36(1.1)$ & $.89^{* *}$ \\
Resolve guilt related to NDE & $1.92(1.1)$ & $.82^{* *}$ \\
Express feelings \& thoughts about NDE & $2.14(0.8)$ & $.73^{* *}$ \\
$\quad$ Validate NDE as real for myself & $2.29(0.9)$ & $.70^{* *}$ \\
Therapist accept NDE & $2.80(1.4)$ & $.58^{*}$ \\
NDE focus of treatment & $2.27(1.2)$ & .34 \\
Therapist aware of NDE & $2.73(1.1)$ & -.02 \\
\hline
\end{tabular}

Note. $n=15 .{ }^{1}$ Therapist Factors: 1 (Not at All) to 4 (Yes). ${ }^{2}$ Treatment Success from 1 (Don't Know/ Not Helpful) to 5 (Yes, Very Helpful). ${ }^{*} p<.05 ;{ }^{* *} p<.01$.

The most commonly endorsed factors were therapist awareness and acceptance of the NDE, with means of 2.73 and 2.80 respectively. The least endorsed factor was that the therapist helped resolve guilt related to the NDE, with a mean of 1.92 .

The relationship between these therapist factors and belief that the psychotherapy was successful in helping them process and integrate their NDE was examined with Pearson's product moment correlations. There were statistically significant positive correlations with large effects between perceived treatment success and all of the therapist factors except whether the therapist was aware of the NDE and the NDE was a focus of treatment. Specifically, a therapist just being aware of NDEs and addressing them in therapy was not sufficient for therapeutic success; rather, having a therapist who accepted the NDE as real and helped the NDEr explore the meaning, express thoughts and feelings, resolve guilt, and validate the NDE was strongly related to reported success in treatment. 


\section{Discussion}

This research evaluated whether a group of 15 adult childhood NDErs who had received psychotherapy found the therapy effective in helping them integrate their NDEs. Reported integration of NDEs was highly related to indicators of wellbeing, with more positive emotions and fewer negative emotions. However, only $27 \%$ of the participants indicated that their psychotherapy supported their NDE integration. The psychotherapy factors that were successful in helping participants process and integrate their NDEs included having therapists who accepted and validated the NDEs as real and who helped NDErs explore the meaning of, express their thoughts and feelings about, and resolve any guilt related to their NDEs.

Thus, the relationship between psychotherapy and psychological integration was somewhat complicated. When psychotherapy was successful, that is, accepting and validating the NDE, supporting expression of feelings and thought, resolving guilt, and exploring the meaning of the NDE, participants reported being more psychologically integrated. However, over $70 \%$ of the participants reported that they found their psychotherapy experiences to be minimally helpful. This finding suggests that the psychological services being delivered to childhood NDErs may need to be refined. The most commonly endorsed psychotherapy factors were that therapists were aware of and accepting that the NDEs were real. Less commonly endorsed were the more specific factors related to how the therapist helped them process and integrate their NDEs. Yet the more specific factors have the strongest relationship with NDErs reporting that the psychotherapy successfully helped them integrate their NDEs. It may be that the psychotherapy process is not leading to the next phase of psychological treatment, which is psychological integration of their experience and aftereffects. When childhood NDErs had successful therapy, psychological integration and emotional wellness were the positive outcomes.

Childhood NDEs can be confusing, which may require therapy to integrate and adjust to the experiences of different phenomena and emotions related to their NDEs. NDErs have identified challenges in communicating their experiences that affect their relationships and coping strategies. Successful clinical support for spiritual and psychological issues might expedite integration sooner than the reported $20+$ years after NDE. These results invite mental health and medical professionals to become educated in the unique attributes of spiritual issues in treatment. 
As is always the case, there are limitations to this study. It was difficult to find childhood NDErs who qualified for the study. The sample size was relatively small, decreasing the reliability of the results and possibly contributing to Type II error whereby results may have been negative not because a relationship did not exist but because the sample size was inadequate to show a relationship that actually existed. It also was difficult to have a wide variation in ethnic diversity of participants, although the sample was diverse with regard to religion, age, education level, and occupations. It is suspected that depending on perceptions of near-death and other spiritually transformative experiences, individuals may not view their experiences as out of the ordinary because the experiences fit in individuals' cultural schemas. More research is needed to examine the relationship between ethnicity and NDEs.

There is a need to develop definitions and empirically validated psychometric instruments for assessing NDE integration. It would be useful to empirically validate the efficacy of psychological interventions and treatment plans to assist with the integration and transcendent stages of psychotherapy. It is suggested that future researchers analyze the effectiveness of different treatment approaches, in particular experiential approaches such as sandtray (Homeyer \& Sweeney, 1998) that supports integration of internal experience. It is imperative future researchers assess which types of treatments to use at certain stages of cognitive development and stages of adjustment to NDEs. For example, a child may need trauma-focused cognitive behavioral therapy or EMDR if the child requires treatment for traumatic circumstances surrounding their NDEs. Many of the participants in this study reported that in therapy they preferred not to focus on the precipitating events but rather on their NDE aftereffects. It is suspected this is the case because the NDE aftereffects are the prominent feature of the current functioning of adults who experienced NDE as a child many decades ago. It is hoped that from these findings of this study, therapeutic treatment programs and interventions can be designed and researched specifically for childhood NDErs.

\section{References}

American Center for the Integration of Spiritually Transformative Experiences. (2010). Retrieved from http://www.aciste.org/index.php/about-stes/what-isan-ste

Atwater, P. M. H. (1996). Children and the near-death phenomenon: Another viewpoint. Journal of Near-Death Studies, 15, 5-16. 
Cohen, J. (1992). A power primer. Psychological Bulletin, 112(1), 155-159.

Diener, E., \& Biswas-Diener, R. (2008). Happiness: Unlocking the mysteries of psychological wealth. New York, NY: Wiley/Blackwell.

Diener, E., Emmons, A., Larsen, R., \& Griffin, S. (1985). The Satisfaction With Life Scale. Journal of Personality Assessment, 49(1), 71-75. Retrieved from http://www.unt.edu/rss/SWLS.pdf

Flanagan, C. (2008). The aftereffects of near-death experiences (Doctoral dissertation). Retrieved from ProQuest Dissertations and Theses database. (AAT3307679)

Foster, R., James, D., \& Holden, J. M. (2009). Practical applications of research on near-death experiences. In J. M. Holden, B. Greyson, \& D. James (Eds.), The handbook of near-death experiences: Thirty years of investigation (pp. 235258). Westport, CT: Praeger/ABC-CLIO.

Gallup, G., \& Proctor, W. (1982). Adventures in immortality: A look beyond the threshold of death. New York, NY: McGraw Hill.

Greyson, B. (1983). The Near-Death Experience Scale: Construction, reliability, and validity. Journal of Nervous and Mental Disease, 171(6), 369-375.

Greyson, B. (1990). Near-death encounters with and without near-death experiences: Comparative NDE Scale profiles. Journal of Near-Death Studies, 8, $151-161$.

Homeyer, L., \& Sweeney, D. (1998). Sandtray: A practical manual. Canyon Lake, TX: Lindan Press.

International Association for Near-Death Studies. (2009). Key facts about neardeath experiences. Retrieved from http://www.iands.org/

International Association for Near-Death Studies. (2010). Building global understanding of near-death experiences. Retrieved from http://www.iands.org/

Lange, R., Greyson, B., \& Houran, J. (2004). A Rasch scaling evaluation of a 'core' near-death experience. British Journal of Psychology, 95, 161-177.

Noyes, R., Fenwick, P., Holden, J. M, \& Christian, S. R. (2009). Aftereffects of pleasurable Western adult near-death experiences. In J. M. Holden, B. Greyson, \& D. James (Eds.), The handbook of near-death experiences: Thirty years of investigation (pp. 41-62). Westport, CT: Praeger/ABC-CLIO.

Seeman, J. (2008). Psychotherapy and the fully functioning person. Bloomington, IN: AuthorHouse.

Sutherland, C. (2009). "Trailing clouds of glory": The near-death experiences of Western children and teens. In J. M. Holden, B. Greyson, \& D. James (Eds.), The handbook of near-death experiences: Thirty years of investigation (pp. 87108). Westport, CT: Praeger/ABC-CLIO. 